Tropical Journal of Pharmaceutical Research January 2021; 20 (1): 183-190

ISSN: $1596-5996$ (print); 1596-9827 (electronic) (C) Pharmacotherapy Group, Faculty of Pharmacy, University of Benin, Benin City, 300001 Nigeria.

\title{
Relationship between adherence to anti-diabetic medication and depression among patients with diabetes mellitus in three selected Chinese hospitals
}

\author{
Haidong Luo ${ }^{1}$, Yin Lin ${ }^{1}$, Jia $\mathrm{Li}^{2}$, Weiguo $\mathrm{Xu}^{3 *}$ \\ ${ }^{1}$ Psychological Counseling and Treatment Center, Hainan Province Anning Hospital, Haikou, Hainan 570200, ${ }^{2}$ Department of \\ Psychology, Hainan People's Hospital, Haikou, Hainan 570311, ${ }^{3}$ Department of Psychiatry, Ningxia Hui Autonomous Region \\ ning' an Hospital (Ningxia Mental Health Center), Yinchuan, Ningxia 750021, China
}

*For correspondence: Email: 21sureger@163.com; Tel/Fax: 0086-0951-2160749

Sent for review: 14 August 2020

Revised accepted: 21 December 2020

\begin{abstract}
Purpose: To determine the relationship between adherence to anti-diabetic medication and depression among patients with diabetes mellitus in three hospitals in Chinese.

Methods: This research utilized a quantitative and descriptive design, and included 200 diabetic patients who fulfilled the inclusion criteria for recruitment through a convenient sampling technique. The study applied Beck's depression inventory II scale for assessment of depression, and a questionnaire for adherence to anti-diabetic medication.

Results: A total of 64 (32\%) participants had diabetes for 6 to 10 years. There was a high level of adherence to anti-diabetic medication in 96 patients (48\%); 74 participants (37\%) had moderate adherence to anti-diabetic medication, while 30 patients (15\%) had low adherence. A majority of the patients (181, $90.5 \%)$ had no depression. Six (6) patients (3\%) had mild mood disturbance, 2 patients $(1 \%)$ had borderline clinical depression, while 11 patients $(5.5 \%)$ experienced moderate depression. Adherence to anti-diabetic medication was not associated with depression $(p=0.068)$. However, depression was associated with age $>50$ years $(p=0.041)$, female sex $(p=0.043)$, long duration of illness (> 5-years) $(p=0.048)$, and presence of one or more comorbidities $(p=0.049)$.

Conclusion: There was no association between adherence to anti-diabetic medication and depression among diabetic patients.
\end{abstract}

Keywords: Adherence to anti-diabetic medication, Beck's depression inventory II, Depression, Diabetes mellitus

\begin{abstract}
This is an Open Access article that uses a fund-ing model which does not charge readers or their institutions for access and distributed under the terms of the Creative Commons Attribution License (http://creativecommons.org/licenses/by/4.0) and the Budapest Open Access Initiative (http://www.budapestopenaccessinitiative.org/read), which permit unrestricted use, distribution, and reproduction in any medium, provided the original work is properly credited.

Tropical Journal of Pharmaceutical Research is indexed by Science Citation Index (SciSearch), Scopus, International Pharmaceutical Abstract, Chemical Abstracts, Embase, Index Copernicus, EBSCO, African Index Medicus, JournalSeek, Journal Citation Reports/Science Edition, Directory of Open Access Journals (DOAJ), African Journal Online, Bioline International, Open-J-Gate and Pharmacy Abstracts
\end{abstract}

\section{INTRODUCTION}

The prevalence of diabetes mellitus (DM), a disease which results in high degree of mortality, is on the increase all over the world [1]. It has been estimated that 300 million people will have
DM by the year 2025, and this figure may reach 439 million in the year 2030 , with a prevalence of $7.7 \%$ of the world population $[2,3]$. It has also been estimated that 3.96 million adult diabetic patients will die of the disease each year, and that diabetes will be responsible for $6.8 \%$ of all 
mortality cases. Depression is a common comorbidity in a chronic disease such as DM. The prevalence of depression is $1.5-2$ times higher in patients with type 2 DM than in type 1 diabetics [1].

Diabetes-induced depression is highly prevalent, with significant adverse impact on life and health [1]. Indeed, the combination of depression and type $2 \mathrm{DM}$ is a public health problem [1, 4, 5]. One very important factor for ensuring effective and efficient management of diabetes is adherence to treatment and compliance with medications [6]. There is a high possibility of depression in DM, and factors such as genetic predisposition and disease duration play important roles in its etiology [7]. The associations among depression, diabetes and non-adherence to anti-diabetes treatment have been reported by physicians for several hundred years [1, 4]. Earlier reports have indicated a frequency of major despair 1.6 - 2 times higher in diabetics than in non-diabetic patients in the general population [8]. Moreover, one out of every 10 diabetic patients is affected with high level depression [9]. The degrees of major lifetime depression in type 2 diabetics are between 24 and $29 \%$ [4], and point prevalence is between 10 and $15 \%$ [10].

Diabetes is a major public health concern, and when complicated with depression, it adversely affects the quality of life and increases mortality of patients [1]. Compared with individuals who have only diabetes, patients with comorbid depression have increased disease burden, greater symptoms severity, increased work disability, and poorer adherence to diet, exercise, and anti-diabetic, lipid-lowering and antihypertensive medications [11].

A descriptive study was conducted to determine the relationship between adherence to antidiabetic medication and depression among DM patients. Moreover, the study was carried out to ascertain the association between various factors and depression, with exclusion of level of adherence to anti-diabetic medication as one of the independent variables.

\section{METHODS}

\section{Ethical approval and consent to participate}

Ethical clearance for the study was received from the ethics committee of Ningxia Hui Autonomous Region Ning'an Hospital Review Board (protocol no. NHACL121420). The purpose of the study was clearly explained to the participants. A written informed consent was obtained from each of them prior to commencement of the study. For participants under 16 years of age, written informed consents were obtained from their parents or guardians. Confidentiality of the responses was assured and maintained throughout the study. The research was conducted in strict adherence to the laws of China and the 2008 Declarations of Helsinki [12].

\section{Study population}

This study was conducted at the Ningxia Hui autonomous region's Ning' an Hospital, Yinchuan, Ningxia, China; The Hainan Province Anning Hospital, Haikou, Hainan, China, and the Hainan People's Hospital, Haikou, Hainan, China. This research was designed in line with descriptive research workflow. Diabetic patients receiving treatment who fulfilled the inclusion criteria during the data collection period were recruited as participants in the study.

\section{Sample size}

Based on prevalence of depression among diabetic patients, the calculated sample size for the study was 200. It was hypothesized that at least $10 \%$ of diabetic patients had mild or higher depression. Moreover, $80 \%$ power $(\beta=0.2), 5 \%$ type-I error $(\alpha=0.05)$, and $95 \%$ of confidence limit were used for sample size calculation. A non-probability convenience sampling technique was employed for choosing the DM patients (diabetic patients were selected based on ease of recruitment).

\section{Sampling criteria}

\section{Inclusion criteria}

The included participants were outpatients who were diagnosed with DM and were receiving treatment at the institutes, DM patients willing to participate in the study, and DM patients able to read Chinese and English languages.

\section{Exclusion criteria}

Patients with acute illnesses and/or psychiatric problem, and patients with any pre-existing depressive disorders, were excluded.

\section{Background variables}

Data regarding socio-demographic variables (age, sex, education, occupation, diet, and exercises) and clinical variables (duration of illness, type of drugs used for treatment, duration of treatment, frequency of drugs, comorbidities (hypertension, bronchial asthma and renal 
failure); family history of diabetes, and current complications) were collected from hospital records of patients.

\section{Diabetic medication adherence questionnaire}

The questionnaire contained 8 items based on the Institutional Protocol for Medical Adherence (not published). The total score was based on patients' responses to eight simple, yes-or-no questions. Yes was scored ' 1 ', while No was scored ' 0 '. High scores indicate high degree of adherence. Scores less than 6 were classified as low adherence, while scores of 6 and 7 indicated medium adherence. A score of 8 was classified as high adherence.

\section{Beck's depression inventory II}

Beck's depression inventory (BDI) II contains 21 multiple-choice, self-report inventory questions. The minimum score obtainable is 0 , while the maximum is 63 . The cutoff for depression is 20 [13]. The scoring of BDI II is as follows: $1-10=$ normal; $11-20$ = mild mood disturbances; 20 25 = borderline clinical depression; 25 - $30=$ moderate depression; 31- $40=$ severe depression, and $>40=$ extreme depression [14].

\section{Statistical analysis}

Statistical analysis was done with SPSS v25.0 IBM Corporation, Armonk, New York, USA. Chisquare test of Independence was used to analyze data. Univariate and multivariate analyses were used to determine the association between background variables and depression. Values of $p<0.05$ were considered statistically significant.

\section{RESULTS}

\section{Study population}

A total of 215 DM patients who were being managed at the institutes declared their willingness to participate in the study. Ten (10) patients had acute illness, while 5 patients had psychiatric problems. These 15 DM patients were excluded from the study. A total of 200 patients with DM were eventually enrolled in the study (Figure 1).

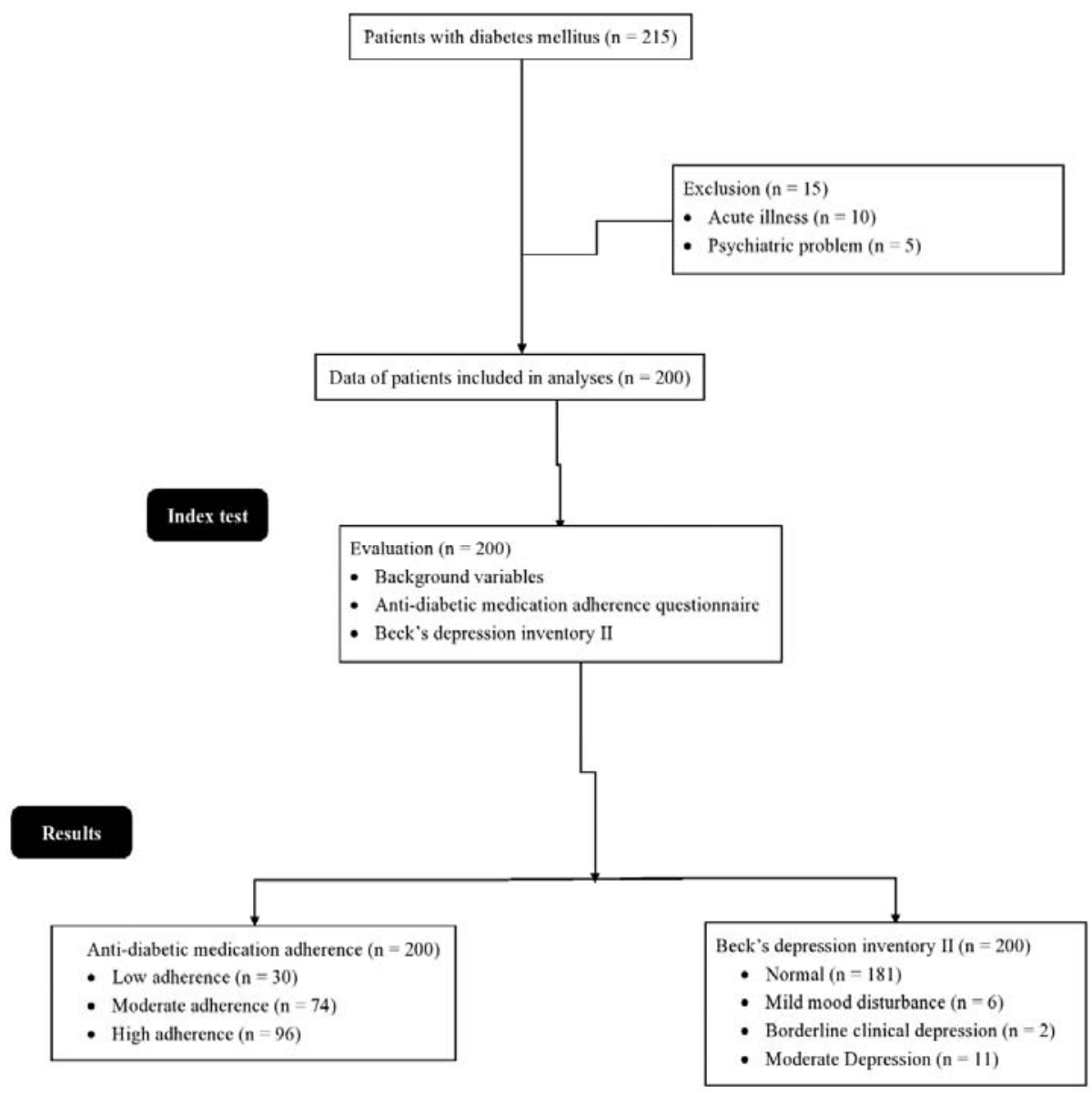

Figure 1: Flow diagram of the study 


\section{Socio-demographic characteristics}

In this study, 73 of the enrolled patients ( $36.5 \%)$ were within the age group of $61-70$ years; 140 (70\%) were female, 72 (36\%) had secondary education, 124 (62\%) were housewives, $160(80$ $\%)$ did not follow diabetic diet, while $143(71.5 \%)$ used regular exercise. These data are shown in Table 1.

Table 1: Socio-demographic characteristics of patients $(n=200)$

\begin{tabular}{lll}
\hline Variable & & Frequency (\%) \\
\hline & $10-20$ & $1(0.5)$ \\
Age & $21-30$ & $3(1.5)$ \\
(years) & $31-40$ & $5(2.5)$ \\
& $41-50$ & $24(12)$ \\
& $51-60$ & $59(29.5)$ \\
& $61-70$ & $73(36.5)$ \\
Sex & $71-80$ & $34(17)$ \\
& $81-90$ & $1(0.5)$ \\
& Male & $60(30)$ \\
& Female & $140(70)$ \\
& No formal education & $67(33.5)$ \\
& Primary education & $46(23)$ \\
& Secondary education & $72(36)$ \\
& Higher education & $7(3.5)$ \\
& Graduate & $5(2.5)$ \\
& Professional & $3(1.5)$ \\
& qualification & $24(12)$ \\
& Retired & $16(8)$ \\
Eccupation & Employed & $33(16.5)$ \\
& Coolie & $124(62)$ \\
& Housewife & $2(1)$ \\
& Politician & $1(0.5)$ \\
& Student & $40(20)$ \\
& Yes & $160(80)$ \\
& No & $57(2.8)$ \\
& No & $143(71.5)$ \\
& Yes &
\end{tabular}

\section{Clinical conditions of the patients}

The study results indicated that 64 participants (32\%) had DM for 6 to 10 years, 146 (73\%) were on oral anti-diabetic drugs, $49(24.5 \%)$ had been on treatment for $6-10$ years, $199(99.5 \%)$ were taking oral hypoglycemic drugs twice a daily, 97 (48.5\%) had hypertension, 137 (68.5 $\%$ ) had no family history of DM, while 179 (89.5 $\%$ ) did not have complications (Table 2).

\section{Responses to questionnaire on adherence to anti-diabetic medication}

Majority of the participants returned positive responses to the items in the adherence questionnaire, indicating adherence to antidiabetes medication. These results are presented in Table 3. The results showed that 96 participants (48\%) had high level of adherence to anti-diabetic medication, 74 participants (37\%) had moderate adherence to anti-diabetic medication adherence, while 30 patients (15\%) had low adherence to anti-diabetic medication. These results are presented in Figure 2.

Table 2: Clinical conditions of patients

\begin{tabular}{|c|c|c|}
\hline Variable & & $\begin{array}{l}\text { Frequency } \\
(\%)\end{array}$ \\
\hline \multirow{9}{*}{ Duration of illness } & $<1$ year & $5(2.5)$ \\
\hline & $1-5$ years & $31(15.5)$ \\
\hline & $6-10$ years & $64(32)$ \\
\hline & $11-15$ years & $48(24)$ \\
\hline & $16-20$ years & $35(17.5)$ \\
\hline & $21-25$ years & $9(4.5)$ \\
\hline & $26-30$ years & $6(3)$ \\
\hline & $31-35$ years & $1(0.5)$ \\
\hline & $36-40$ years & $1(0.5)$ \\
\hline \multirow{4}{*}{$\begin{array}{l}\text { Route of the } \\
\text { antidiabetic drugs }\end{array}$} & Oral & $146(73)$ \\
\hline & Injectable & $1(0.5)$ \\
\hline & Oral \& Injectable & $43(21.5)$ \\
\hline & $<1$ year & $5(2.5)$ \\
\hline \multirow{7}{*}{$\begin{array}{l}\text { Duration of } \\
\text { treatment }\end{array}$} & $1-5$ years & $31(15.5)$ \\
\hline & $6-10$ years & 63 (31.5) \\
\hline & $11-15$ years & $49(24.5)$ \\
\hline & $16-20$ years & $35(17.5)$ \\
\hline & $21-25$ years & $8(4)$ \\
\hline & $26-30$ years & $6(3)$ \\
\hline & $31-35$ years & $2(1)$ \\
\hline \multirow{4}{*}{$\begin{array}{l}\text { Frequency of the } \\
\text { drugs }\end{array}$} & $36-40$ years & $1(0.5)$ \\
\hline & Twice in a day & $199(99.5)$ \\
\hline & Thrice in a day & $1(0.5)$ \\
\hline & Hypertension & $97(48.5)$ \\
\hline \multirow{5}{*}{ Comorbidities } & Bronchial asthma & $5(2.5)$ \\
\hline & Hypertension \& & $1(0.5)$ \\
\hline & Renal failure & \\
\hline & $\begin{array}{l}\text { Hypertension \& } \\
\text { bronchial asthma }\end{array}$ & $2(1)$ \\
\hline & $\begin{array}{l}\text { No comorbid } \\
\text { illness }\end{array}$ & $95(47.5)$ \\
\hline \multirow{2}{*}{$\begin{array}{l}\text { Family history of } \\
\text { diabetes }\end{array}$} & Yes & $63(31.5)$ \\
\hline & No & $137(68.5)$ \\
\hline \multirow{4}{*}{$\begin{array}{l}\text { Current } \\
\text { complication }\end{array}$} & Neuropathy & $14(7)$ \\
\hline & Retinopathy. & $7(3.5)$ \\
\hline & Nephropathy & $0(0)$ \\
\hline & No complication & $179(89.5)$ \\
\hline
\end{tabular}

\section{Incidence of depression among patients}

Beck's depression inventory II was used to determine the percentage distribution of depression among the DM patients. The results in Figure 3 show that most of the respondents $(181,90.5 \%)$ did not have depression. Only 6 participants $(6 \%)$ had mild mood disturbance, while the numbers of patients with borderline clinical depression and moderate depression were $2(1 \%)$ and $11(5.5 \%)$, respectively.

\section{Risk factors for depression}

Table 4 shows that there was no association between adherence to anti-diabetic medication and depression among DM patients $(p=0.068)$. 
Table 3: Responses to anti-diabetic medication adherence questionnaire

\begin{tabular}{lll}
\hline $\mathbf{S} / \mathbf{N}$ & Item & Frequency (\%) \\
\hline 1 & Did not forget to take medication & $137(68.5)$ \\
& Forgot to take medication & $63(31.5)$ \\
2 & Did not make mistake in medication & $151(75.5)$ \\
& Mistake in medication & $49(24.5)$ \\
3 & Did not stop taking medication & $176(88)$ \\
& Stopped taking medication & $24(12)$ \\
4 & Did not forget to take medication while traveling & $88(44)$ \\
& Forgot to take medication while traveling & $127(63.5)$ \\
5 & Took medication on the previous day & $73(36.5)$ \\
& Did not take medication on the previous day & $177(88.5)$ \\
6 & Did not stop the medication when symptoms were under control & $23(11.5)$ \\
& Stopped medication when symptoms were under control & $163(81.5)$ \\
7 & Taking medication is convenient & $37(18.5)$ \\
& Taking medication is inconvenient & $172(86)$ \\
8 & Did not experience difficulty in remembering to take medication & $28(14)$ \\
\hline
\end{tabular}

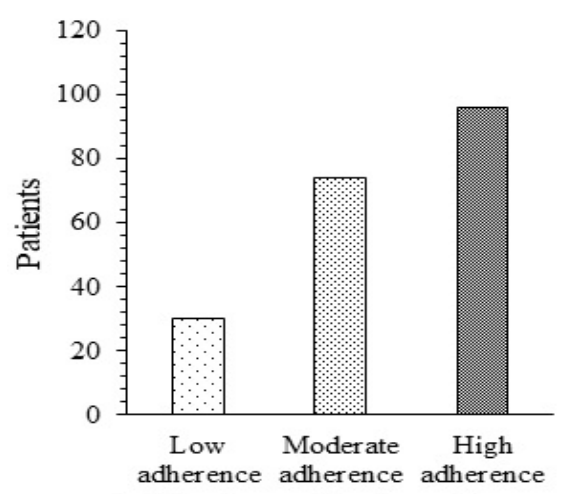

Anti-diabetic medication adherence

Figure 2: Degrees of patients' adherence to antidiabetic medication

Univariate analysis showed that depression was associated with age $>50$ years $(p=0.021)$, female sex ( $p=0.023)$, absence of exercise $(p=$ 0.026 ), long duration of illness ( $>5$ years) ( $p=$ 0.029 ), and presence of one or more comorbid

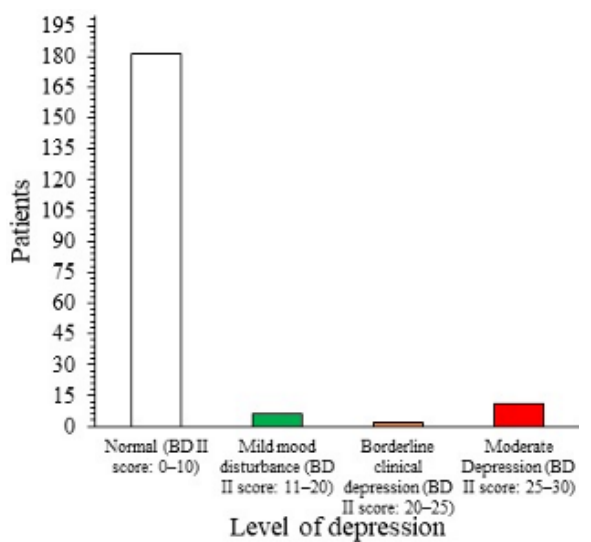

Figure 3: Levels of depression in enrolled patients illnesses ( $p=0.042)$. In addition, multivariate analysis revealed that depression was associated with age $>50$ years, female sex, long duration of illness ( $>5$ years), and presence of comorbidities (Table 5).

Table 4: Association of depression with anti-diabetic medication adherence

\begin{tabular}{lcccc}
\hline \multirow{2}{*}{ Drug compliance } & \multicolumn{2}{c}{ Depression } & & \multirow{2}{*}{ Chi-square } \\
\cline { 2 - 3 } & Normal & Depression & & \\
\cline { 2 - 3 } Number of included patients in study & 187 & 13 & \multirow{2}{*}{5.391} & \multirow{2}{*}{0.068} \\
\hline Low adherence & 30 & 0 & \\
Medium adherence & 72 & 3 & & \\
High adherence & 85 & 10 & & \\
\hline
\end{tabular}

Table 5: Association of depression with background variables among participants

\begin{tabular}{lccc}
\hline Variable & Odds ratio & 95\% confidence interval & $\boldsymbol{P}$ \\
\hline Age $(>50$ years $v s<50$ years) & 1.234 & $0.469-0.985$ & 0.041 \\
Sex (female $^{*}$ vs male) & 1.567 & $0.567-0.875$ & 0.043 \\
Exercises (no vs yes) $_{\text {Duration of illness }\left(>5 \text { years }{ }^{*} \text { vs }<5 \text { years) }\right.}$ & 0.896 & $0.442-0.887$ & 0.056 \\
Comorbid illness (presence $^{*}$ vs absence) & 1.256 & $0.354-0.987$ & 0.048 \\
\hline
\end{tabular}

An odds ratio more than $1(p<0.05)$ was considered significant. Data of normal patients $(n=181)$ were considered as reference standard. "Significant, i.e., associated with depression 


\section{DISCUSSION}

The study found that $146(73 \%)$ patients were on oral anti-diabetic drugs once or twice daily. Majority of the patients preferred treatment with oral hypoglycemic drugs. There was high level of medication adherence with oral medication [15]. Thus, physicians are advised to prescribe oral hypoglycemic agents for DM patients so as to ensure high level of adherence to anti-diabetic medication. It was found that 48,37 and $15 \%$ of the patients had high, moderate, and low adherence, respectively, to anti-diabetic medication. These findings are similar to those obtained earlier in a retrospective cohort study [15] and in cross-sectional studies [16,17]. In these studies, the diabetic patients had high level of adherence to anti-diabetic medication.

Majority of the participants did not have depression. The findings of this study are in agreement with results obtained in a crosssectional study [4] and in a community-based study [18] which revealed that there was no association between anti-diabetic medication adherence and depression in diabetic patients.

In this study, age < 50 years, female sex, long duration of illness ( $>5$ years), and the presence of one or more comorbid illnesses were associated with depression. These are independent factors for depression in diabetes patients irrespective of adherence to anti-diabetic medications [19]. The results are consistent with findings in similar studies $[4,17,19]$. In this study, 105 patients $(52.5 \%)$ had comorbid illnesses. Comorbidity is major complication in DM patients [19]. Thus, it is recommended that physicians should consider comorbidities while treating DM patients.

\section{Limitations of the study}

There are several limitations in this study. For example, it was a retrospective study, and it lacked randomized trial. The cutoff for depression in BDI II was 20. The BDI was shaped by Aaron T Beck which is a 21 multiplechoice-question, self-report inventory. This inventory is the most widely used psychometric test for measurement of severity of depression. There are three versions of the BDI, first published in 1961 and later revised in 1978. The $\mathrm{BDI}$ is widely used as an assessment tool by health care professionals and researchers in a variety of settings, but the value of cutoff varies from country to country [14]. In China, the cutoff for medical samples is 20 [20]. The Morisky adherence questionnaire is a clinical research method used for assessing adherence of patients to medication [21]. The Morisky scale is a validated medication non-adherence assessment tool. This scale has already been cited in over 70 articles since its publication in 1986, and has since then been validated. However, it was not used in the current study. Although the validated Morisky scale is used to assess the risk of medication non-adherence, there is no single measure accepted as a 'gold standard' for evaluating medical adherence [16]. Therefore, the present study did not use the Morisky adherence questionnaire for medication adherence evaluation among DM patients. There is lack of clarity regarding the assessment of treatment compliance. This should have been assessed using blood glucose levels, HbA1c levels, and the development of complications of diabetes.

Previous studies have shown that depression in patients with diabetes affects the ability for selfcare, including dieting, exercise, keeping medical appointments, blood glucose monitoring, and foot care. However, these vital factors were not evaluated in the current study. The internal consistency of each factor and the general scale of BDI II were not assessed using Cronbach's $\alpha$ coefficient. Comorbid medical illnesses and current diabetic complications may be confounding factors associated with depression in diabetic patients, but they were not included in the simple logistic regression analysis.

\section{CONCLUSION}

There is no association between adherence to anti-diabetic medication and depression in diabetic patients in the hospitals studied. Old age, female sex, long duration of illness, and presence of one or more comorbidities were independent risk factors for depression among diabetic patients. It is hereby recommended that the relevant authorities publish an institutional protocol for medical adherence for diabetic patients.

\section{DECLARATIONS}

\section{Acknowledgement}

Authors are thankful to the medical and nursing staff of Ningxia Hui Autonomous Region Ning' An Hospital, Yinchuan, Ningxia, China, The Hainan Province Anning Hospital, Haikou, Hainan, China, and the Hainan People's Hospital, Haikou, Hainan, China. No funding was received for this work. 


\section{Availability of data and materials}

The datasets used and analyzed during the current study available from the corresponding author on reasonable request.

\section{Conflict of interest}

The authors declare that they have no conflict of interest or any other competing interest regarding this research.

\section{Authors' contributions}

We declare that this work was done by the authors named in this article and all liabilities pertaining to claims relating to the content of this article will be borne by the authors. All authors read and approved the manuscript for publication. Hidong Luo and Yin Lin contributed equally to resources, literature review, data curation, supervision, the investigation, formal analysis, and validation of the study. Jia Li was a project administrator, contributed to conceptualization, literature review, resources, formal analysis, and data curation of the study. Wiguo $\mathrm{Xu}$ contributed to the methodology, literature review, and resources of the study, draft, and edited the manuscript for intellectual content. All authors agree to be accountable for all aspects of work ensuring integrity and accuracy.

\section{Open Access}

This is an Open Access article that uses a funding model which does not charge readers or their institutions for access and distributed under the terms of the Creative Commons Attribution License (http://creativecommons.org/licenses/by/ 4.0) and the Budapest Open Access Initiative (http://www.budapestopenaccessinitiative.org/rea d), which permit unrestricted use, distribution, and reproduction in any medium, provided the original work is properly credited.

\section{REFERENCES}

1. Egede LE, Ellis C. Diabetes and depression: Global perspectives. Diabetes Res Clin Pract 2010; 87(3): 302312.

2. Guariguata L, Whiting DR, Hambleton I, Beagley J, Linnenkamp U, Shaw JE. Global estimates of diabetes prevalence for 2013 and projections for 2035. Diabetes Res Clin Pract 2014; 103(2): 137-149.

3. Farag YM, Gaballa MR. Diabesity: An overview of a rising epidemic. Nephrol Dial Transplant 2011; 26(1): 28-35.
4. El-Shafie TM, El-Saghier EO, Ramadan IK. Depression Among type 2 Diabetic patients. Egyptian J Hosp Med 2011; 44: 258-271.

5. Daniele $T M$, de Bruin VM, e Forte $A C$, de Oliveira DS, Pompeu CM, de Bruin PF. The relationship between physical activity, restless legs syndrome, and healthrelated quality of life in type 2 diabetes. Endocrine 2013; 44(1): 125-131.

6. Awodele O, Osuolale JA. Medication adherence in type 2 diabetes patients: Study of patients in Alimosho General Hospital, Igando, Lagos, Nigeria. Afr Health Sci 2015; 15(2): 513-522.

7. Atta A, Kachela B, Arif JM, Tahir KB, Shoukat N, Ali NB. Assessment of medication adherence and knowledge regarding the disease among ambulatory patients with diabetes mellitus DM in Karachi, Pakistan. J Young Pharm 2015; 7(4): 328-340.

8. Markowitz SM, Gonzalez JS, Wilkinson JL, Safren SA. A review of treating depression in diabetes: Emerging findings. Psychosomatics 2011; 52(1): 1-8.

9. Pouwer F, Geelhoed Duijvestijn PH, Tack CJ, Bazelmans E, Beekman AJ, Heine RJ, Snoek FJ. Prevalence of comorbid depression is high in out-patients with Type 1 or Type 2 diabetes mellitus. Results from three outpatient clinics in the Netherlands. Diabet Med 2010; 27(2): 217-224.

10. Fisher L, Skaff MM, Mullan JT, Arean P, Glasgow R, Masharani U. A longitudinal study of affective and anxiety disorders, depressive affect and diabetes distress in adults with Type 2 diabetes. Diabet Med 2008; 25(9): 1096-1101.

11. Nguyen AL, Green J, Enguidanos S. The relationship between depressive symptoms, diabetes symptoms, and self-management among an urban, low-income Latino population. J Diabetes Complications 2015; 29(8): 1003-1008.

12. Declaration of Helsinki (V2008). [Cited 2020 November 1]. Available from: http://www.chictr.org.cn/uploads/ documents/201610/df3df4b49abd489e9eabf5d02e2bad 45.pdf

13. Zhu $M$, Liang $H$, Feng J, Wang Z, Wang $W$, Zhou $Y$. Effectiveness of medical self-experience counseling in pregnant women after echocardiographic detection of a suspected fetal heart anomaly: A longitudinal study. J Obstet Gynaecol Res 2016; 42(10): 1236-1244.

14. Stechova $K$, Mastikova $L$, Urbaniec $K$, Vanis $M$, Hylmarova S, Kvapil M, Pastor Z. Sexual dysfunction in women treated for type 1 diabetes and the impact of coexisting thyroid disease. Sex Med 2019; 7(2): 217226.

15. Huber CA, Reich O. Medication adherence in patients with diabetes mellitus: does physician drug dispensing enhance quality of care? Evidence from a large health claims database in Switzerland. Patient Prefer Adherence 2016; 10: 1803-1809.

16. Jimmy B, Jose J, Al-Hinai ZA, Wadair IK, Al-Amri GH. Adherence to medications among type 2 diabetes mellitus patients in three districts of Al Dakhliyah

Trop J Pharm Res, January 2021; 20(1): 189 
Governorate, Oman: A cross-sectional pilot study. Sultan Qaboos Univ Med J 2014; 14(2): e231-e235.

17. Wang J, Bian RW, Mo YZ. Validation of the Chinese version of the eight-item Morisky medication adherence scale in patients with type 2 diabetes mellitus. Journal of Clinical Gerontology \& Geriatrics 2013; 4: 119-122.

18. Aminu AS, Chandrasekaran V, Nair S. Depression among patients with diabetes: A community-based study in south India. J Med Sci 2017; 37(6): 237-244.

19. Kayar Y, Kayar NB, Erden SC, Onem R, Ekinci I, Emegil S, Ismayilova M, Ozmen A, Baysal B, Danalioglu A. The relationship between depression and demographic risk factors, individual lifestyle factors, and health complications in patients with type 2 diabetes mellitus. Biomed Res 2017; 28(4): 1560-1565.

20. Wang YP, Gorenstein C. Psychometric properties of the Beck Depression Inventory-II: A comprehensive review. Braz J Psychiatry 2013; 35(4): 416-431.

21. Wong MC, Wu CH, Wang HH, Li HW, Hui EM, Lam AT, Chung RY, Yip BH, Morisky DE. Association between the 8-item Morisky medication adherence scale (MMAS8) score and glycaemic control among Chinese diabetes patients. J Clin Pharmacol 2015; 55(3): 279-287. 